\title{
Case Sensitive
}

National Cancer Institute

\section{Source}

National Cancer Institute. Case Sensitive. NCI Thesaurus. Code C71490.

Indicates that a text matching operation disting uishes upper-case (capital) letters from lower case. 